\title{
Two ultracold atoms in a completely anisotropic trap
}

\author{
Jun-Jun Liang ${ }^{1,2}$,Chao Zhang ${ }^{1}$ \\ ${ }^{1}$ Department of Physics, Shanxi University, Taiyuan 030006, China \\ ${ }^{2}$ Institute of Theoretical Physics, Shanxi University, Taiyuan 030006, China
}

\begin{abstract}
As a limiting case of ultracold atoms trapped in deep optical lattices, we consider two interacting atoms trapped in a general anisotropic harmonic oscillator potential, and obtain exact solutions of the Schrödinger equation for this system. The energy spectra for different geometries of the trapping potential are compared.
\end{abstract}

PACS number(s): 34.50. s, 32.80.Pj

Recently optical lattice becomes a convenient tool to study many-body physics in periodical potential, and the physics of quantum degenerate atomic gases trapped in an optical lattice have been intensively investigated theoretically $1,2,3,4$ and experimentally ${ }^{5,6,7}$. The characteristic of short-range interaction between atoms makes optical lattice ideal to experimentally realize Bose (or Fermi) Hubbard models. In deep optical lattice multiband Hubbard models are extremely difficult to handle. One can gain some insight into this system by neglecting tunneling between adjacent sites. In this case, Bloch bands are nearly flat, and an individual well may be approximated by a harmonic oscillator potential. As a result, we model the lattice as an array of microscopic harmonic traps, each of which is occupied with a few atoms. A precise understanding of atoms interacting in this simple model is a prerequisite for analysis of manybody physics in optical lattices with resonantly enhanced interactions. We only consider the physics of two identical atoms now, instead of different atoms that may feel different trapping frequencies, which leads to a coupling of center-of-mass (CM) and relative motion ${ }^{6}$.

The system of two interacting atoms in a harmonic trap has been studied analytically both in spherically $\underline{6.8}$ and axially symmetric cases ${ }^{9}$. The interaction is described in terms of an s-wave pseudopotential by the regularized $\delta$ - function ${ }^{10}$. Higher partial wave, such as p-wave is also considered 11 . In this paper we investigate the completely anisotropic harmonic potential and obtain the exact solutions for two interacting atoms confined in a trap with three different frequencies. At last, we compare the properties of energy spectra for trapping potential of different geometries.

We consider two interacting atoms with identical mass $m$, which are confined in a completely anisotropic harmonic trapping potential with three frequencies $\omega_{x}, \omega_{y}$ and $\omega_{z}$. The Hamiltonian can be written as

$H=-\frac{\hbar^{2}}{2 m} \nabla_{1}^{2}-\frac{\hbar^{2}}{2 m} \nabla_{2}^{2}+V_{\text {Trap }}\left(\mathbf{r}_{1}\right)+V_{\text {Trap }}\left(\mathbf{r}_{2}\right)+V_{\text {int }}\left(\mathbf{r}_{1}-\mathbf{r}_{2}\right)$,

where $\mathbf{r}_{1}$ and $\mathbf{r}_{2}$ denote the positions of two atoms respectively, and $V_{\text {Trap }}(\mathbf{r})$ is the completely anisotropic harmonic trapping potential

$$
V_{\text {Trap }}(\mathbf{r})=\frac{1}{2} m\left(\omega_{x}^{2} x^{2}+\omega_{y}^{2} y^{2}+\omega_{z}^{2} z^{2}\right) .
$$

In the ultracold regime, atomic interaction is dominated by $s$-wave scattering. In this case, the interaction potential is modeled by a pointlike form which is expressed in terms of the so-called regularized $\delta$-function

$$
V_{\text {int }}(\mathbf{r})=\frac{4 \pi a_{0} \hbar^{2}}{m} \delta(\mathbf{r}) \frac{\partial}{\partial r} r,
$$

where $r=|\mathbf{r}|$ denotes the distance between two atoms and $a_{0}$ the scattering length.

For convenience, in the following calculations we use dimensionless variables, in which lengths are expressed in units of $\sqrt{\hbar / m \omega_{x}}$ and energies are expressed in units of $\hbar \omega_{x}$. In addition, all frequencies are denoted with $\omega_{x}$ by introducing parameters $\eta_{y}$ and $\eta_{z}$, where $\eta_{y}=\omega_{y} / \omega_{x}$ and $\eta_{z}=\omega_{z} / \omega_{x}$. In this two-body system, as the Hamiltonian (11) has a quadratic form for both kinetic energy and harmonic oscillator potential, the motion of $\mathrm{CM}$ and relative one can be decoupled by introducing $\mathbf{r}=\left(\mathbf{r}_{1}-\mathbf{r}_{2}\right) / \sqrt{2}$ and $\mathbf{R}=\left(\mathbf{r}_{1}+\mathbf{r}_{2}\right) / \sqrt{2}$, where $\mathbf{R}$ is the CM coordinate and $\mathbf{r}$ the relative coordinate. The eigenfunctions and eigenvalues of CM Hamiltonian $H_{C M}=-\frac{1}{2} \nabla_{R}^{2}+V_{C M}(\mathbf{R})$, where $V_{C M}(\mathbf{R})=\left(X^{2}+\right.$ $\left.\eta_{y}^{2} Y^{2}+\eta_{z}^{2} Z^{2}\right) / 2$, are the solutions of three-dimension harmonic oscillator, which are tackled analytically in the standard textbook of quantum mechanics.

Now we consider the relative motion of the system, of which the Hamiltonian $H_{r e l}$ reads

$$
H_{r e l}=-\frac{1}{2} \nabla^{2}+V_{r e l}(\mathbf{r})+\sqrt{2} \pi a_{0} \delta(\mathbf{r}) \frac{\partial}{\partial r} r,
$$

where $V_{\text {rel }}(\mathbf{r})=\left(x^{2}+\eta_{y}^{2} y^{2}+\eta_{z}^{2} z^{2}\right) / 2$. The solutions can be determined by the following Schrödinger equation

$$
H_{r e l} \Psi=E \Psi .
$$

To obtain the eigenvalues and eigenfunctions of the Hamiltonian (4) is our main tasks in this paper. First let us consider two non-interacting atoms in the same trapping potential, and the system satisfies the following equation

$$
\begin{array}{r}
{\left[-\frac{1}{2} \nabla^{2}+\frac{1}{2}\left(x^{2}+\eta_{y}^{2} y^{2}+\eta_{z}^{2} z^{2}\right)\right] \Phi_{n_{x} n_{y} n_{z}}(x, y, z)} \\
=E_{n_{x} n_{y} n_{z}} \Phi_{n_{x} n_{y} n_{z}}(x, y, z),
\end{array}
$$


with $\Phi_{n_{x} n_{y} n_{z}}(x, y, z)=\Phi_{n_{x}}(x) \Phi_{n_{y}}\left(\sqrt{\eta_{y}} y\right) \Phi_{n_{z}}\left(\sqrt{\eta_{z}} z\right)$, in which $\Phi_{n_{w}}(\alpha w)=N_{n_{w}} e^{-\frac{1}{2} \alpha^{2} w^{2}} H_{n_{w}}(\alpha w)$ denotes the eigenfunctions of 1D harmonic oscillator, where the constant $N_{n_{w}}=\left(\alpha / \sqrt{\pi} 2^{n_{w}}\left(n_{w}\right) !\right)^{1 / 2}$ with parameter $\alpha=1$, $\sqrt{\eta_{y}}, \sqrt{\eta_{z}}$ and corresponding variable $w=x, y, z$, while $H_{n_{w}}(\alpha w)$ is the Hermite function. The dimensionless eigenenergies of the system are $E_{n_{x} n_{y} n_{z}}=\left(n_{x}+1 / 2\right)+$ $\eta_{y}\left(n_{y}+1 / 2\right)+\eta_{z}\left(n_{z}+1 / 2\right)$. We use complete set $\left\{\Phi_{n_{x} n_{y} n_{z}}(x, y, z)\right\}$ to expand the unknown wavefunction $\Psi$ in Eq. (5),

$$
\Psi(\mathbf{r})=\sum_{n_{x}, n_{y}, n_{z}} c_{n_{x} n_{y} n_{z}} \Phi_{n_{x} n_{y} n_{z}}
$$

By inserting (4) and (7) into (5), the Schrödinger equation can be express as

$$
\begin{aligned}
& \sum_{n_{x}, n_{y}, n_{z}} c_{n_{x} n_{y} n_{z}}(\left.E_{n_{x} n_{y} n_{z}}-E\right) \Phi_{n_{x} n_{y} n_{z}}+\sqrt{2} \pi a_{0} \delta(r) \\
& \times \frac{\partial}{\partial r} r \sum_{n_{x}, n_{y}, n_{z}} c_{n_{x} n_{y} n_{z}} \Phi_{n_{x} n_{y} n_{z}}=0 .
\end{aligned}
$$

To determine the expansion coefficients $c_{n_{x} n_{y} n_{z}}$ we project Eq.(8) onto state $\Phi_{m_{x} m_{y} m_{z}}$ with nonnegative integers $m_{x}, m_{y}$ and $m_{z}$, and obtain the structure of $c_{m_{x} m_{y} m_{z}}$

$$
c_{m_{x} m_{y} m_{z}}=C \frac{\left[\Phi_{m_{x}}(0) \Phi_{m_{y}}(0) \Phi_{m_{z}}(0)\right]^{*}}{E_{m_{x} m_{y} m_{z}}-E}
$$

where $C$ is a constant that is related to

$C=-\sqrt{2} \pi a_{0}\left[\frac{\partial}{\partial r}\left(r \sum_{n_{x}, n_{y}, n_{z}} c_{n_{x} n_{y} n_{z}} \Phi_{n_{x} n_{y} n_{z}}(x, y, z)\right)\right]_{r \rightarrow 0}$

Substituting the expression (9) for coefficient $c_{n_{x} n_{y} n_{z}}$ into Eq. (10), the constant $\mathrm{C}$ is removed and we have an equation which denotes the relation between the scattering length and eigenenergies

$$
\sqrt{2} \pi\left[\frac{\partial}{\partial r}\left(r \Psi_{E}(x, y, z)\right)\right]_{r \rightarrow 0}=-\frac{1}{a_{0}}
$$

where

$$
\begin{aligned}
& \Psi_{E}(x, y, z)= \\
& \sum_{n_{x}, n_{y}, n_{z}} \frac{\left[\Phi_{n_{x}}(0) \Phi_{n_{y}}(0) \Phi_{n_{z}}(0)\right]^{*} \Phi_{n_{x}}(x) \Phi_{n_{y}}(y) \Phi_{n_{z}}\left(\gamma_{2}^{2}\right)}{E_{n_{x} n_{y} n_{z}}-E}
\end{aligned}
$$

is the non-normalized eigenstates of the Schrödinger equation (5). Using the expressions of eigenfunctions and eigenvalues of Eq.(6) we find the form of wavefunction

$$
\begin{gathered}
\Psi_{E}(x, y, z)=e^{-\frac{1}{2}\left(x^{2}+\eta_{y} y^{2}+\eta_{z} z^{2}\right)} \sum_{n_{x}, n_{y}, n_{z}} N_{n_{x}}^{2} N_{n_{y}}^{2} N_{n_{z}}^{2} \\
\times \frac{H_{n_{x}}(x) H_{n_{x}}(0) H_{n_{y}}\left(\sqrt{\eta_{y}} y\right) H_{n_{y}}(0) H_{n_{z}}\left(\sqrt{\eta_{z}} z\right) H_{n_{z}}(0)}{n_{x}+\eta_{y} n_{y}+\eta_{z} n_{z}+f(E)}(13)
\end{gathered}
$$

where we let $f(E)=\frac{1}{2}+\frac{1}{2} \eta_{y}+\frac{1}{2} \eta_{z}-E$ for convenience and the denominator $n_{x}+\eta_{y} n_{y}+\eta_{z} n_{z}+f(E)$ denotes the shift of energy.

By utilizing integral representation

$\frac{1}{n_{x}+\eta_{y} n_{y}+\eta_{z} n_{z}+f(E)}=\int_{0}^{1} d q q^{n_{x}+\eta_{y} n_{y}+\eta_{z} n_{z}+f(E)-1}$,

which is valid for $f(E)>0$ since $n_{x}+\eta_{y} n_{y}+\eta_{z} n_{z} \geqslant 0$, the wavefunction is transformed into

$$
\begin{aligned}
& \Psi_{E}(x, y, z)=\sum_{n_{x}, n_{y}, n_{z}} \frac{\left(\eta_{y} \eta_{z}\right)^{1 / 2}}{\pi^{3 / 2}} e^{-\frac{1}{2}\left(x^{2}+\eta_{y} y^{2}+\eta_{z} z^{2}\right)} \\
& \int_{0}^{1} d q q^{f(E)-1} \times\left[q^{n_{x}} \frac{H_{n_{x}}(x) H_{n_{x}}(0)}{2^{n_{x}} n_{x} !}\right] \\
& \times\left[q^{\eta_{y} n_{y}} \frac{H_{n_{y}}\left(\sqrt{\eta_{y}} y\right) H_{n_{y}}(0)}{2^{n_{y}} n_{y} !}\right]\left[q^{\eta_{z} n_{z}} \frac{H_{n_{z}}\left(\sqrt{\eta_{z}} z\right) H_{n_{z}}}{2^{n_{z}} n_{z} !}(1) .5\right)
\end{aligned}
$$

The limitation $f(E)>0$ effectively means that we restrict the following steps to energies smaller than the noninteracting harmonic oscillator ground state energy. For higher energies, analytic continuation may be used. The series given by the product of two Hermite functions with variable $v$ can be simplified via following formula

$$
\begin{aligned}
\sum_{n=0}^{\infty} & \frac{H_{n}(z) H_{n}\left(z_{1}\right)}{n !} v^{n} \\
& =\frac{1}{\sqrt{1-4 v^{2}}} \exp \left(\frac{2 v\left(2 v\left(z^{2}+z_{1}^{2}\right)-2 z z_{1}\right)}{4 v^{2}-1}\right)
\end{aligned}
$$

which is valid for $|v|<1 / 2$. In the expression (15) $v=$ $q / 2$ satisfies the condition when the integral variable $q$ changes from 0 to 1 . The eigenfunction can be obtained in integral representation with (16)

$$
\begin{aligned}
& \Psi_{E}(x, y, z)=\frac{\left(\eta_{y} \eta_{z}\right)^{1 / 2}}{2 \pi^{3 / 2}} e^{-\frac{1}{2}\left(x^{2}+\eta_{y} y^{2}+\eta_{z} z^{2}\right)} \int_{0}^{\infty} d t e^{-u t} \\
& \times \frac{\exp \left(\frac{x^{2} e^{-t}}{e^{-t}-1}\right)}{\sqrt{1-e^{-t}}} \frac{\exp \left(\frac{\eta_{y} y^{2} e^{-\eta_{y} t}}{e^{-\eta_{y} t}-1}\right)}{\sqrt{1-e^{-\eta_{y} t}}} \frac{\exp \left(\frac{\eta_{z} z^{2} e^{-\eta_{z} t}}{e^{-\eta_{z} t}-1}\right)}{\sqrt{1-e^{-\eta_{z} t}}}
\end{aligned}
$$

where we introduce new variables $t$ and $u$, which are defined as $q^{2}=e^{-t}, u=\frac{f(E)}{2}$. 
Due to the pointlike force of two atoms, it is necessary to check the behavior of the wavefunction for small $x, y$ and $z$. When $x, y, z \rightarrow 0$, the main contribution for (17) is dominated by small $t$. It is reasonable to perform the approximation $e^{-\lambda t}=1-\lambda t$ for $\lambda=1, \eta_{y}, \eta_{z}$ in the wavefunction and neglect the influence of energy in the leading order. After some straightforward algebra, the wavefunction for small $t$ can be written as

$$
\begin{aligned}
\Psi_{E}(x, y, z) & \approx \frac{1}{2 \pi^{3 / 2}} \int_{0}^{\infty} d t \frac{\exp \left[-\left(x^{2}+y^{2}+z^{2}\right) / t\right]}{t^{3 / 2}} \\
& =\frac{1}{2 \pi\left(x^{2}+y^{2}+z^{2}\right)^{1 / 2}}, \quad x, y, z \rightarrow 0
\end{aligned}
$$

which gives no contribution to (11), and the divergent factor $t^{-3 / 2}$ in the integral above can be subtracted from the wavefunction $\Psi_{E}(x, y, z)$. Using the fact that

$$
\begin{aligned}
\frac{\partial}{\partial r}[r g(x, y, z)]= & g(x, y, z)+x \frac{\partial g(x, y, z)}{\partial x}+y \frac{\partial g(x, y, z)}{\partial y} \\
& +z \frac{\partial g(x, y, z)}{\partial z}
\end{aligned}
$$

with $g(x, y, z)$ denoting an analytic function, it can be verified easily that

$$
\left[\frac{\partial}{\partial r}\left(r \Psi_{E}(r)\right)\right]_{r \rightarrow 0}=\left[\Psi_{E}(r)\right]_{x, y, z \rightarrow 0}
$$

Combining (17) and (20), we obtain

$$
\begin{aligned}
& {\left[\frac{\partial}{\partial r} r\left(\Psi_{E}(x, y, z)\right)\right]_{r \rightarrow 0}=\frac{\left(\eta_{y} \eta_{z}\right)^{1 / 2}}{2 \pi^{3 / 2}}} \\
& \left.\times \int_{0}^{\infty} d t\left[\frac{e^{-u t}}{\sqrt{1-e^{-t}} \sqrt{1-e^{-\eta_{y} t}} \sqrt{1-e^{-\eta_{z} t}}}-\frac{1}{t^{3 / 2}}\right] 21\right)
\end{aligned}
$$

Substituting the expression (21) into (11), the eigenenergies takes the form of implicit function

$$
Z(u)=-\frac{\sqrt{2 \pi}}{a_{0}}
$$

where

$$
Z(u)=\int_{0}^{\infty} d t\left[\frac{\left(\eta_{y} \eta_{z}\right)^{1 / 2} e^{-u t}}{\sqrt{1-e^{-t}} \sqrt{1-e^{-\eta_{y} t}} \sqrt{1-e^{-\eta_{z} t}}}-\frac{1}{t^{3 / 2}}\right] .
$$

When the trap is axially symmetric, let $\eta_{y}=\eta_{z}=\eta$ and (22) reduces to
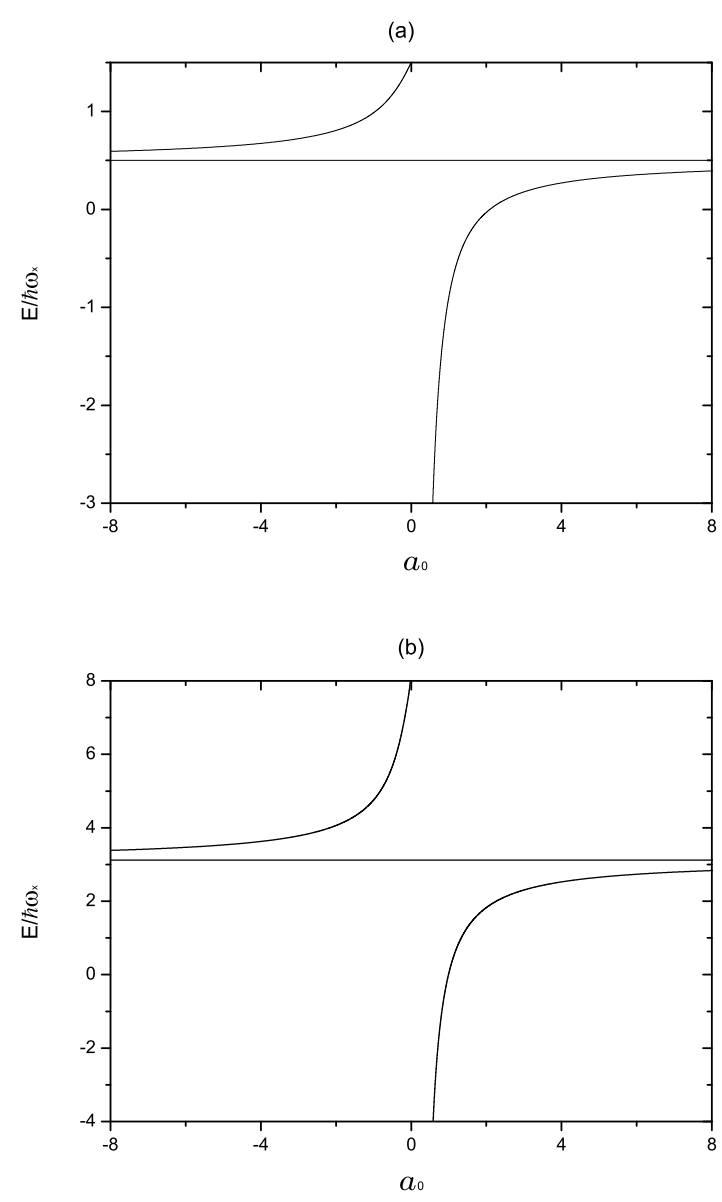

FIG. 1: Eigenenergies of relative motion for the system of two atoms interacting via s-wave pseudopotential and confined in a harmonic trap with parameters (a) $\eta_{y}=1, \eta_{z}=1$ (b) $\eta_{y}=5, \eta_{z}=10$. The scattering length $a_{0}$ is scaled in the units of $\sqrt{\hbar / m \omega_{x}}$.

$$
\int_{0}^{\infty} d t\left[\frac{\eta e^{-u t}}{\sqrt{1-e^{-t}}\left(1-e^{-\eta t}\right)}-\frac{1}{t^{3 / 2}}\right]=-\frac{\sqrt{2 \pi}}{a_{0}},
$$

which is the result given in 9 . The additional factor $\sqrt{2}$ on the right side is due to different choice of the relative and $\mathrm{CM}$ coordinates.

For the isotropic case, by substituting $\eta_{y}=\eta_{z}=1$ into (22) the result agrees with the well-known relation between eigenenergies and scattering length in $\underline{8}$

$$
\sqrt{2} \frac{\Gamma(u)}{\Gamma\left(u-\frac{1}{2}\right)}=\frac{1}{a_{0}} .
$$

In the case of $f(E)<0$, the eigenenergies are generally determined by numerical method and we limit our discussion to analytic regime of $f(E)>0$ (i.e. $u>0$ ). 
Fig.1 shows the energy spectra of two interacting ultracold atoms confined in harmonic traps for (a) isotropic case of $\eta_{y}=1, \eta_{z}=1$ and (b) completely anisotropic case of $\eta_{y}=5, \eta_{z}=10$. When $a_{0}=0$, the interaction disappears, and the pole of $Z(u)$ presents the energy of two atoms, which is corresponding to the ground-state eigenenergy of the harmonic oscillator with non-interacting case, and this can be verified easily by (12), (20), (21) and (22). In the isotropic case, the non-interacting ground state energy is $E=3 / 2$, and $E=16 / 2$ in the fully anisotropic case. In the case of $a_{0}<0$, the energy level is shifted downwards, while the scattering exhibits an attractive feature. On the contrary, for $a_{0}>0$ the energy approaches $1 / a_{0}^{2}$ while interactions are repulsive. For the two unitary limits of $a_{0} \rightarrow-\infty$ and $a_{0} \rightarrow+\infty$, they have the same asymptotic values which become larger with the increase of $\eta_{y}$ and $\eta_{z}$, and this is due to the zero of $Z(u)$. It is obvious that there is no pole of $Z(u)$ for $u>0$. We note that by choosing larger ratios of frequencies, the energy level moves upwards.

In summary, we have presented the analytical solutions for the system of two ultracold atoms interacting via $s$-wave pseudopotential in the completely anisotropic harmonic trap. For special ratios of frequencies, we obtained the results both in spherically symmetric case ${ }^{8}$ and axially symmetric one ${ }^{9}$. With the model developed in this paper, the system of two atoms interacting via s-wave pseudopotential can be studied in any shape of the harmonic trap. Our theoretical result shows a clear physical picture and can be used in many-body physics of ultracold atoms trapped in the optical lattice.

This work was supported by the Youth Science Foundations of Shanxi Province and Shanxi University under Grants Nos. 2006021002, and by the National Science Foundation of China under Grants Nos. 10444002.
1 D.B.M. Dickerscheid, U. Al Khawaja, D.van Oosten, H.T.C. Stoof, Phys. Rev. A 71, 043604 (2005).

2 Arnaud Koetsier, D. B. M. Dickerscheid, and H. T. C. Stoof, Phys. Rev. A 74, 033621 (2006).

${ }^{3}$ K. B. Gubbels, D. B. M. Dickerscheid, and H. T. C. Stoof, New J. Phys. 8, 151 (2006).

${ }^{4}$ Roberto B. Diener and Tin-Lun Ho, Phys. Rev. Lett. 96, 010402 (2006).

5 Thilo Stödferle et al., Phys. Rev. Lett. 96, 030401 (2006).

${ }^{6}$ F. Deuretzbacher et al., cond-mat/0703322.

7 C. Ospelkaus et al., Phys. Rev. Lett. 97, 120402 (2006); C.
Ospelkaus, Ph.D. thesis, University at Hamburg (2006).

8 T. Busch, B.-G. Englert, K. Rzazewski, and M. Wilkens, Found. Phys. 28, 549 (1998).

9 Z. Idziaszek and T. Calarco, Phys. Rev. A 71, 050701 (2005); 74, 022712 (2006).

10 K. Huang and C. N. Yang, Phys. Rev. 105, 767 (1957); K. Huang, Statistical Mechanics (Wiley, New York, 1963).

11 R. Stock, A. Silberfarb, E. L. Bolda, and I. H. Deutsch, Phys. Rev. Lett. 94, 023202 (2005); Z. Idziaszek and T. Calarco, Phys. Rev. Lett. 96, 013201 (2006). 